\title{
Influence de la phosphatation au zinc sur la résistance a la corrosion d'un acier au carbone en milieu marin
}

\author{
A. Ettaqi ${ }^{1,{ }^{*}}$, A. Irhzo' ${ }^{1}$ R. Drissi Daoudi' et B. Chaker ${ }^{2}$ \\ 'Laboratoire de Corrosion et de Protection \\ ${ }^{2}$ Laboratoire des Sciences des Matériaux, Université Hassan II, Faculté des Sciences Aîn \\ Chock, route d'Eljadida, BP 5366 Maârif, Casablanca, Maroc.
}

(Reçu le 22 Juin 2005, accepté le 15 Septembre 2005)

*Correspondance et tirés à part, e-mail : ettaqi2003@yahoo.fr

\section{Résumé}

Dans le cadre de cette étude, nous avons montré que l'allure des courbes cathodiques du métal non phosphaté, plaide en faveur d'un processus cinétique essentiellement contrôlé par une diffusion pure. La vitesse de corrosion, aussi bien celle déterminée à partir des courbes cathodiques que celle obtenue par dosage colorimétrique du fer passé en solution, augmente lorsque la vitesse de rotation de l'électrode augmente. Ceci conforte l'hypothèse d'un contrôle diffusionnel. La phosphatation de l'électrode ne semble pas modifier l'allure des courbes cathodiques. Toutefois, la hauteur du palier de diffusion dépend de l'épaisseur de la couche de phosphatation et prend des valeurs inférieures à celles de l'acier non revêtu pour une épaisseur de la couche de phosphatation supérieure à deux micromètres. La couche de phosphatation renforce notablement la résistance à la corrosion de la couche interne, composée essentiellement d'oxydes de fer, par la diminution de la vitesse de corrosion au fur et à mesure que l'épaisseur de la couche croît. Néanmoins, l'effet bénéfique de la phosphatation est maximum pour une épaisseur de trois micromètres. Cette constatation se manifeste également lors du comportement anodique.

Mots-clés: Phosphatation au zinc, corrosion, acier XC N10, milieu marin (NaCl 3\%).

\section{Abstract}

Zinc phosphatation influence on the resistance to corrosion of a carbonbased steel in marine medium

In the frame of the present investigation, we have showed that the profile of the cathodic curves of a non phosphated metal, plead in favour to a kinetic profile essentially monitored by a pure diffusion. The corrosion rate, obtained from the cathodic curves as 
well as by a colorimetrical dosage of the dissolved iron, increases when the rotation speed of the electrode increases. Which show a diffusional control the phosphatation of the electrode does not seem to modify the cathodic curves profile. However the height of the diffusion palier depends on the thickness of the phosphatation layer. It takes values lower than those of an uncovered steel for a layer thickness of two micrometers the phosphatation layer reinforce notably the resistance to the corrosion of the internal layer which is essentially composed with iron oxides, by decreasing the corrosion rate with the increase of the layer. However, the benefical effect of the phosphatation is maximal for a thickness of three micrometers. This effect occurs also during the anodic behaviour.

Keywords : Phosphatation to the zinc, corrosion, steel XC N10, marine medium (NaCl 3\%)

\section{Introduction}

La protection contre la corrosion de nombreux biens manufacturés métalliques et de produits de grande consommation, supposent l'application d'une couche de peinture ou de vernis. Cette application ne peut se concevoir sans l'étape intermédiaire d'une mise en condition du matériau par la préparation de surface et la conversion chimique. Ce sont les procédés de phosphatation qui ont le plus fortement contribué à cette conversion chimique de surface et ils n'ont jamais cessé d'évoluer pour répondre aux exigences de plus en plus grandes des industriels [1-4]. Dans tous les cas, la couche de phosphatation qui assure la cohésion entre le revêtement final et l'acier, doit elle-même adhérer parfaitement à l'acier. Cette condition implique à l'évidence que l'opération de phosphatation doit être réalisée sur un métal sain, c'est-à-dire dépourvue de toutes les souillures provenant de :

- sa préparation: ce sont en général des traitements thermiques qui provoquent l'oxydation à chaux de l'acier. Les oxydes ainsi obtenus sont nommés II calamine II ;

- son stockage: l'humidité ambiante peut, dans de nombreux cas, déclencher un phénomène d'oxydation à froid bien connu II la roville II ;

- sa manutention ou son utilisation préalable : ce sont les produits gras provenant de l'emboutissage, la protection temporaire, de la manutention par des engins mécaniques plus au moins sales. L'appellation des ces souillures est rassemblée sous le nom de "graisses I. La préparation de surface, qui précède le stade de phosphatation proprement dit, consiste donc à éliminer dans l'ordre : les graisses par l'opération de dégraissage et la roville et la calamine par l'opération de décapage.

Lorsqu'on réussit à obtenir une surface du métal parfaitement propre, celle-ci reste hétérogène (rugosités, différences de structures cristallines, etc. ), qui constituent un 
départ à la corrosion électrochimique. On diminue le risque en transformant la surface du métal par phosphatation. On obtient ainsi une couche plus homogène chimiquement, plus inerte et nettement plus favorable à l'accrochage des peintures. On distingue deux types de phosphatation: la phosphatation microcristalline (dite au zinc) et la phosphatation amorphe ou passivation.

Parmi les méthodes d'essais les plus fréquemment utilisées pour tester la résistance à la corrosion d'un matériau, nous avons utilisé l'essai au brouillard salin [5], qui a pour but de déterminer, selon une procédure normalisée (ASTM B 117-63) le degré de corrosion du l'acier soumis à des conditions de pression, de température, de concentration et de pH biens déterminées. Les résultats obtenus ont montré que la qualité de protection est notablement supérieure pour une phosphatation au Zinc que pour une phosphatation amorphe.

Plusieurs chercheurs ont montré le rôle de la phosphatation sur la résistance à la corrosion des aciers $[6,7]$. Il ressort de ces études que si la couche de phosphatation doit servir de base à une peinture, la dimension des cristaux est déterminante. La phosphatation d'un métal résulte de plusieurs processus s'effectuant simultanément: une attaque acide du métal, dont la cinétique est favorisée par la présence d'oxydants dans la solution et un déplacement des équilibres d'hydrolyse des phosphates acides et formation de phosphates tri métalliques insolubles.

Toutefois les auteurs [8-10] sont partagés quand aux mécanismes de ces processus. Selon W. MACHU [11], la précipitation de phosphates tri métalliques a lieu sur les zones micro cathodiques. D'autres auteurs, tels que W.I. WULFSON et al [12], V. CUPR et J.B. PELIKAN [13-16], émettent l'hypothèse de la précipitation des phosphates sur les zones micro anodiques. Ces auteurs, à la suite d'une série d'observations, proposent la formation d'une couche amorphe sur les sites anodiques. Cette couche, composée de phosphates mixtes de Zinc et de Fer, serait la base du développement des germes cristallins de phosphates tri métalliques. Leur raisonnement se base sur l'existence d'anions complexes $\left(\mathrm{Zn}^{\mathrm{PO}} \mathrm{O}_{4}\right)$ issus de la réaction de dissolution du dihydrogénophosphate de Zinc [17-19]. Selon W. MACHU ; M. GEBHARDT, quel que soit le procédé de phosphatation, la couche de conversion chimique se compose d'une partie interne au contact du métal constitué d'oxyde de fer et de phosphate de fer. Elle comporte en outre, une partie externe cristalline qui, dans le cas de la phosphatation au Zinc, se compose essentiellement de phosphate mixte Fer-Zinc. La partie interne constitue une véritable couche de passivation du métal alors que la partie externe ne constitue qu'une barrière physique à la pénétration de l'électrolyte [20].

Nous proposons dans le cadre de ce travail, d'étudier l'impact de la phosphatation au zinc, notamment son épaisseur sur la résistance à la corrosion d'un acier XC N10 dans l'eau de mer, équivalente à une solution de chlorure de sodium $3 \%$. 


\section{Matériaux utilisés et méthodes d'étude}

L'acier retenu dans notre étude est l'acier au carbone XC N10 (Norme AFNOR). L'analyse chimique (Tableav 1) par spectrométrie d'émission UV donne la composition en $\%$ de poids des éléments existants :

Tableau 1 : Composition chimique de l'acier XC NIO

\begin{tabular}{|l|l|l|l|l|l|}
\hline C & Si & S & P & Mn & Ni \\
\hline 0.1074 & 0.1589 & 0.0213 & 0.0241 & 0.5493 & 0.0222 \\
\hline
\end{tabular}

\begin{tabular}{|l|l|l|l|l|l|l|}
\hline $\mathbf{C r}$ & $\mathbf{C u}$ & $\mathbf{S n}$ & $\mathbf{C o}$ & $\mathbf{A l}$ & $\mathbf{P b}$ & $\mathbf{N b}$ \\
\hline 0.0208 & 0.044 & 0.0045 & 0.114 & 0.0094 & 0.0035 & 0.0013 \\
\hline
\end{tabular}

Les éprouvettes destinées à l'étude gravimétrique se présentent sous forme des rectangles. Elles ont été dégraissées, rincées, phosphatées, rincées et cuites dans une étuve à $100^{\circ} \mathrm{C}$ pendant 30 minutes. Les électrodes destinées à l'étude électrochimique se présentent sous forme d'un cylindre de $1.766 \mathrm{~cm}^{2}$ de section, revêtu d'une gaine thermo rétractable constituant ainsi l'électrode de travail à disque tournant. Avant chaque essai, ces électrodes ont subi un dégraissage, un rinçage, une phosphatation, un rinçage puis une cuisson dans une étuve à $100^{\circ} \mathrm{C}$ pendant 30 minutes.

L'électrolyte utilisé est une solution de chlorure de sodium à $3 \%$ en poids qui est sensiblement équivalente du point de vue force ionique à l'eau de mer. Le bain de phosphatation est une solution diluée contenant des éléments anioniques $\left(\mathrm{H}_{2} \mathrm{PO}_{4}{ }^{-}\right)$et cationiques $\left(\mathrm{Zn}^{2+}, \mathrm{Fe}^{2+}\right)$ capables de réagir avec la surface métallique conduisant à la formation des cristaux sur cette surface. Les principaux constituants sont :

- l'acide phosphorique libre $\mathrm{H}_{3} \mathrm{PO}_{4}$;

- le phosphate mono zincique $\mathrm{Zn}\left(\mathrm{H}_{2} \mathrm{PO}_{4}\right)_{2}$;

- l'accélérateur $\mathrm{NaNO}_{2}$.

En effet, trois types de phosphatation peuvent exister :

- phosphatation amorphe au Fer (vivianite ou phosphophilite);

- phosphatation microcristalline au Zinc (hopéite ou phosphophilite);

- phosphatation microcristalline Zn - Ni (phosphophilite ou phosphonicolite).

L'étude micrographique des trois types de phosphatation est présentée sur les Photos I, 2 et 3. 


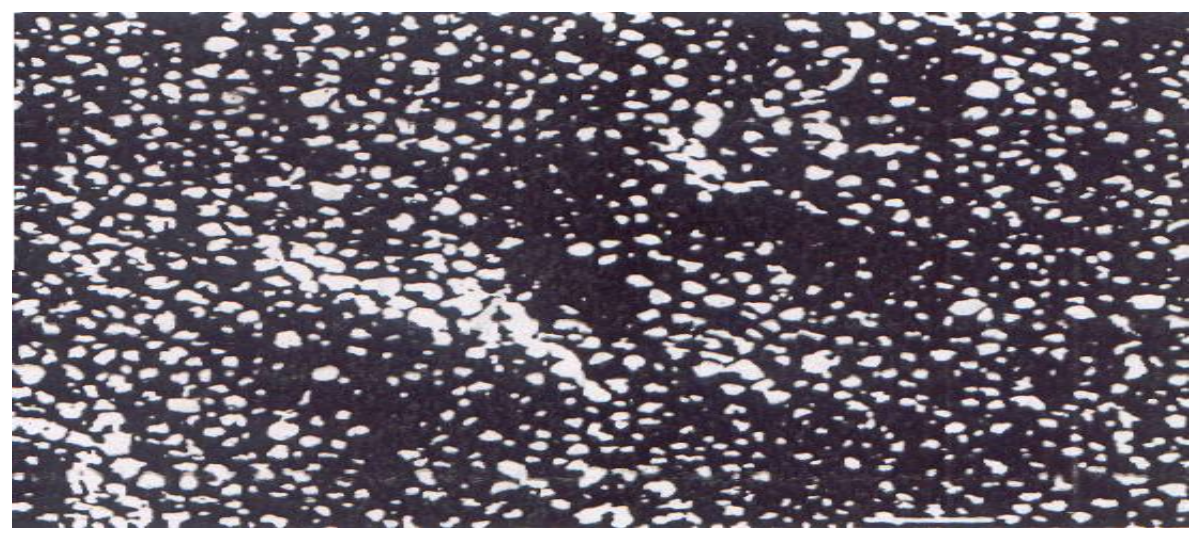

Photo 1 : Phosphatation amorphe au Fer de l'acier utilisé $(G=800)$

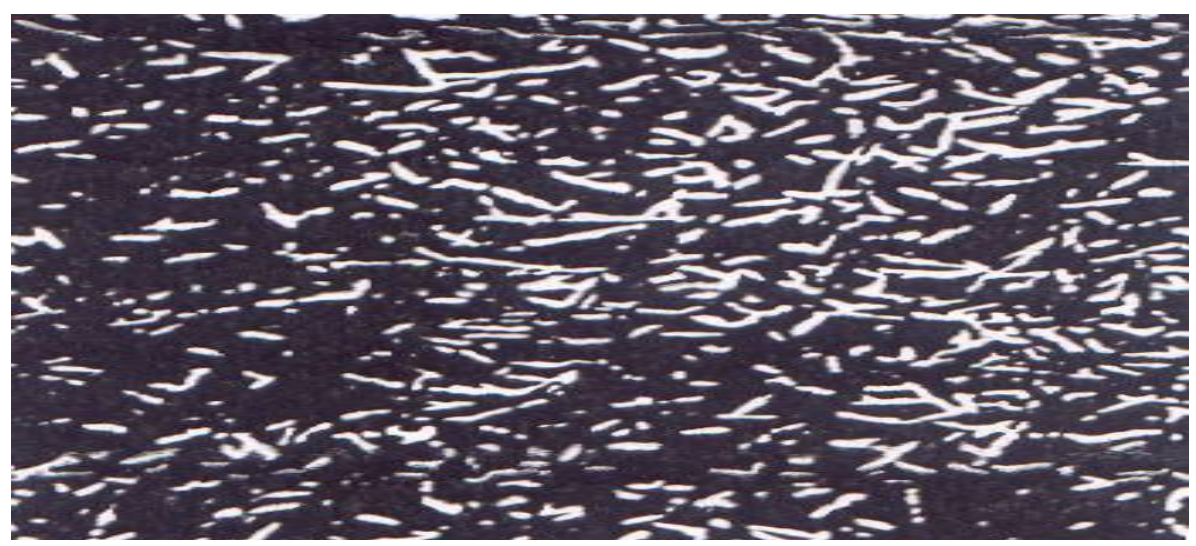

Photo 2: Phosphatation microcristalline au Zinc de l'acier utilisé $(G=800$

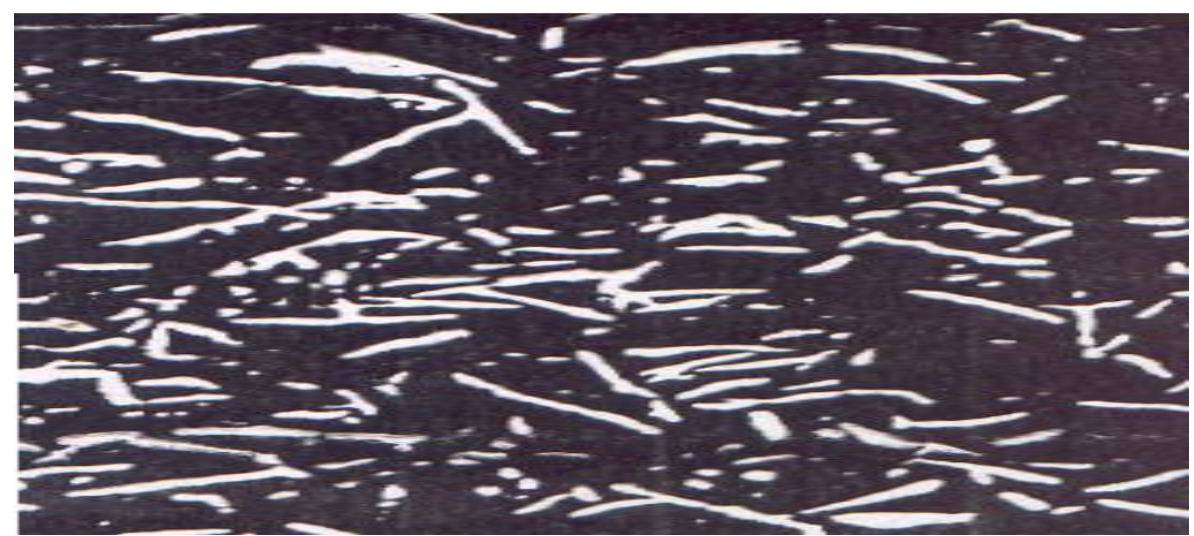

Photo 3 : Phosphatation microcristalline $Z n-N i$ de l'acier utilisé $(G=800)$ 
La concentration totale des différents produits est suffisamment faible pour obtenir une bonne ionisation du bain. L'addition de l'accélérateur, communément des oxydants minéraux, permet de réduire considérablement le temps de la réaction. Le bain comporte également des éléments " affineurs " qui permettent la précipitation des minuscules particules sur l'acier. Chacune de ces particules constituera le germe à partir du quel les cristaux de phosphates pourront se former puis se développer.

La concentration du fer passé en solution a été réalisée par deux types d'essais : - des mesures des pertes de poids en pesant les éprouvettes phosphatées avant et après immersion dans la solution de $\mathrm{NaCl} 3 \%$.

- des dosages du fer passé en solution par absorption atomique type SPECTR AA-20 et par colorimétrique (spectrophotomètre UV - Visible type VARIAN 90 à double faisceau). La surface de l'échantillon étudié est débarrassée des produits de corrosion par attaque au moyen de l'acide chlorhydrique. Les solutions à doser ont été chauffées en présence de quelques gouttes d'acide chlorhydrique concentré afin de dissoudre complètement les oxydes de fer. Ensuite, elles sont refroidies et filtrées. Les ions ferriques éventuellement formés sont réduits par une solution à $10 \%$ de chlorhydrate d'hydroxylamine. Une solution à $0.5 \%$ de chlorhydrate d'orthophénantroline est ensuite ajoutée pour former un complexe rouge.

L'étude électrochimique a été réalisée par un ensemble TACUSSEL composé d'un potentiostat, d'un enregistreur de courant et d'un millivoltmètre électronique à très haute impédance d'entrée. La cellule d'essai est constituée d'un récipient dont lequel sont aménagées les orifices permettant l'introduction d'une électrode de référence au calomel saturée, d'une contre électrode au platine et de l'électrode de travail. Ces électrodes sont reliées à un ensemble TACUSSEL. Le test au brouillard salin consiste à faire subir à un échantillon une attaque corrosive accélérée par un milieu salin artificiel de composition bien définie, dans des conditions de pression et de température. La solution aqueuse saline est constituée de chlorure de sodium à $5 \%$ en masse dont le pH est fixé à $7 \%$. La solution est pulvérisée dans une chambre de pulvérisation par de l'air comprimé. Ce dernier est envoyé au pulvérisateur sous une pression de 1 bar avec une humidité relative de 85 à $90 \%$. La température est de $35^{\circ} \mathrm{C}$.

La disposition des pulvérisateurs et des déflecteurs et la conception géométrique de la chambre de pulvérisation sont tels que la solution saline pulvérisée ne se dépose que sous l'effet de la pesanteur sur l'échantillon à tester. Le degré de la résistance à la corrosion est apprécié, soit par le nombre de piqûres par unité de surface, soit par la surface totale des tâches, soit par comparaison à un échantillon témoin. 


\section{Résultats et discussion}

\section{3-1. Etude gravimétrique}

La Figure 1 représente la perte en poids en fonction du temps d'immersion à diverses épaisseurs de la couche de phosphatation. On constate un accroissement de la perte en poids avec le temps d'immersion.

L'évolution de la perte en poids en fonction de l'épaisseur de la couche de phosphate, résumé par la Figure 2, montre une décroissance de la variation de masse lorsque l'épaisseur augmente donc lorsque l'épaisseur de la couche de phosphate croît.

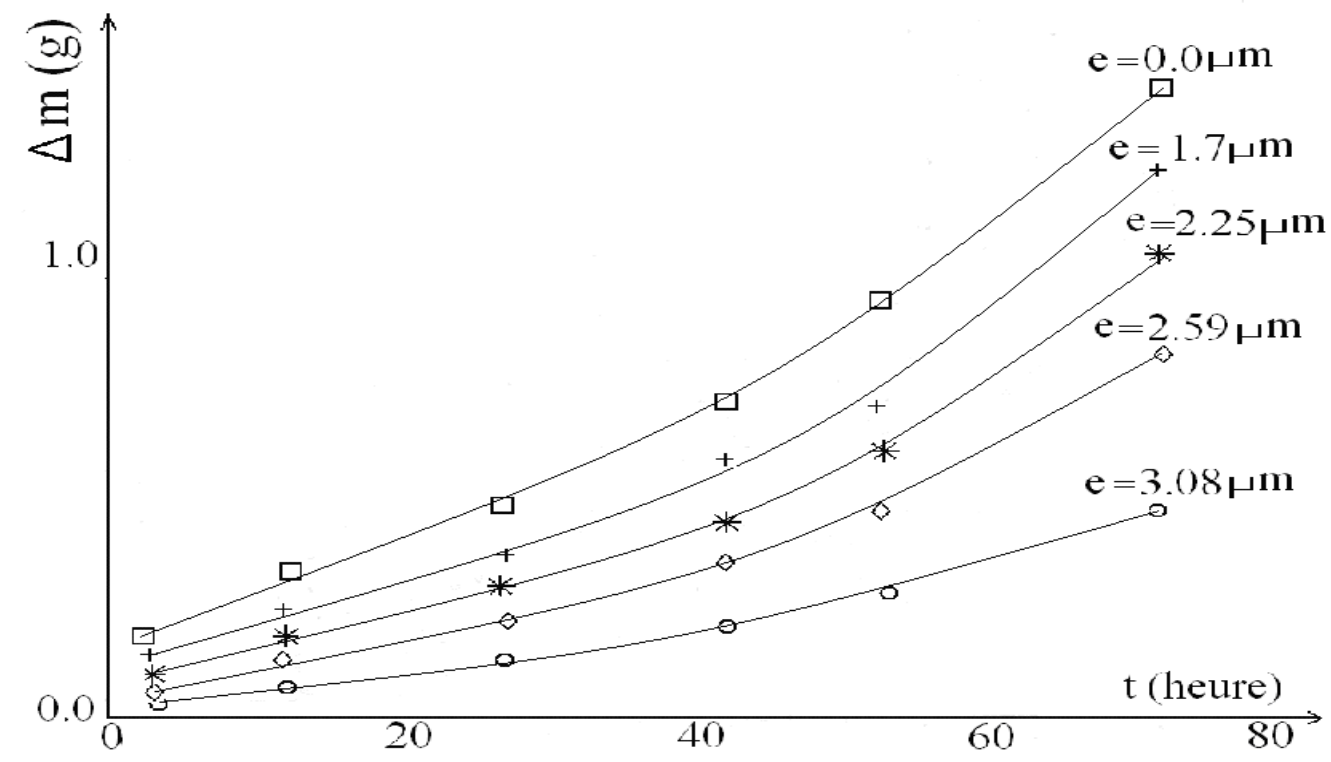

Figure 1 : Perte de poids des éprouvettes en fonction du temps d'immersion dans $\mathrm{NaCl} 3 \%$ à diverses épaisseurs de la couche de phosphatation

De plus les observations des éprouvettes faites immédiatement après immersion des solutions de chlorures de sodium $3 \%$ nous amènent à conclure que la corrosion est uniforme et que dans ces conditions, la densité de courant de corrosion est reliée à la perte de masse m par la loi de Faraday :

$$
i_{\text {cor }}=(n F / M) \cdot(m / t)
$$

où $n$ représente le nombre d'électrons échangés dans la réaction d'oxydation et $t$ le temps de maintien dans la solution électrolytique.

Le test de la résistance à la corrosion après six heures au brouillard salin des éprouvettes phosphatées, a permis de mettre en évidence le rôle de l'épaisseur de la couche de phosphate. 


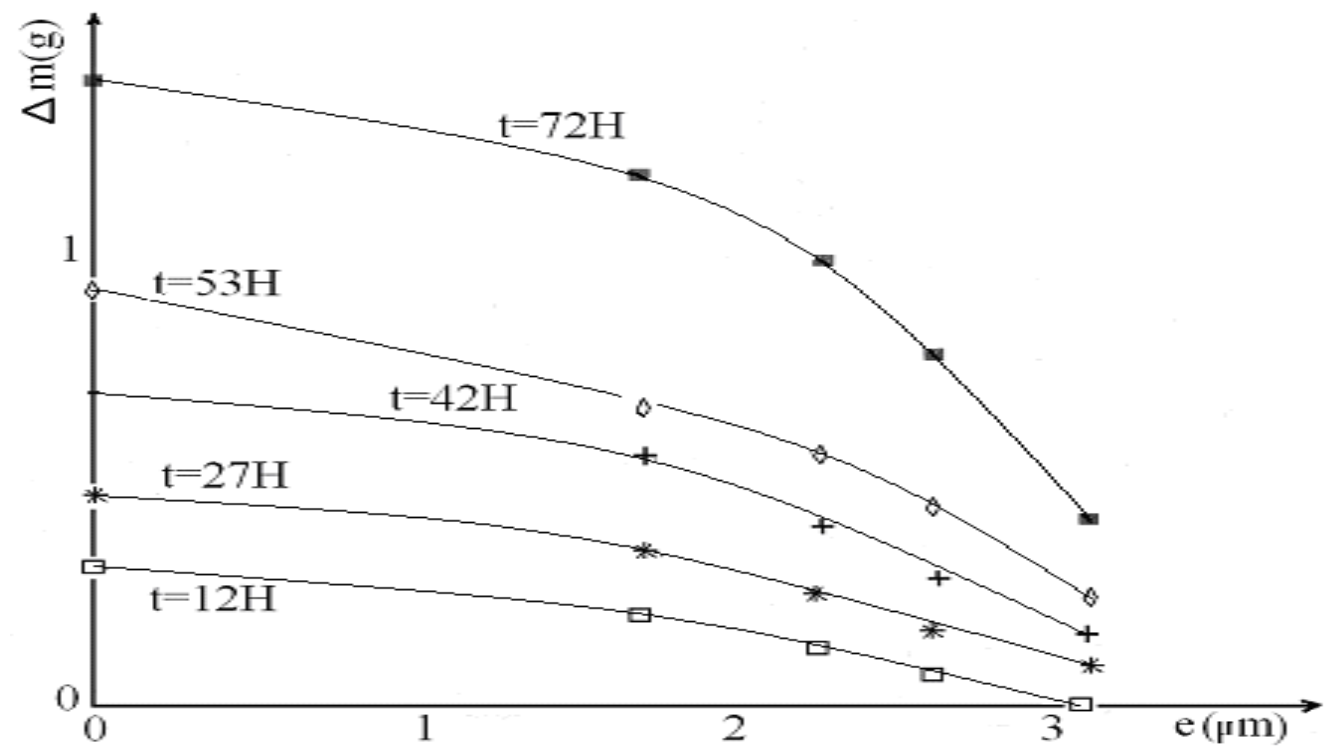

Figure 2 : Perte de poids des éprouvettes en fonction de la couche de phosphatation à divers temps d'immersion dans $\mathrm{NaCl} 3 \%$

Les valeurs de la densité du courant de corrosion déduites de la loi de Faraday sont représentées dans le Tableau 2. On constate que les vitesses de corrosion, obtenues par dosage colorimétrique sont en bon accord avec celles déterminées par analyse spectrophotométrie. L'augmentation de l'épaisseur de la couche de phosphate se traduit par une diminution de la vitesse de corrosion.

Tableau 2 : Valeurs de la densité du courant de corrosion déduites de la loi de Faraday

\begin{tabular}{|c|c|c|c|c|c|c|c|c|}
\hline $\begin{array}{c}\mathrm{N}^{\circ} \\
\text { Eprou- } \\
\text { vette }\end{array}$ & $\begin{array}{l}\text { Surface } \\
\left(\mathrm{cm}^{2}\right)\end{array}$ & $\begin{array}{l}\text { Epais- } \\
\text { seur } \\
(\mu \mathrm{m})\end{array}$ & $\begin{array}{l}\text { Variation } \\
\text { de } \\
\text { masse } \\
\text { (g) }\end{array}$ & $\begin{array}{c}\text { Durée } \\
\text { d'attaque } \\
\text { t (heure) }\end{array}$ & $\begin{array}{l}\text { Dosage } \\
\text { Par A.A } \\
\text { (mg/L) }\end{array}$ & $\begin{array}{c}\text { Dosage } \\
\text { Par } \\
\text { Colorimé- } \\
\text { trie } \\
\text { (mg/L) }\end{array}$ & $\begin{array}{c}\mathrm{i}_{\mathrm{cor}} \\
\text { Par } \\
\text { Absorption } \\
\text { atomique } \\
\left(\mu \mathrm{A} / \mathrm{cm}^{2}\right) \\
\end{array}$ & $\begin{array}{c}\mathbf{i}_{\text {cor }} \\
\text { Par } \\
\text { Colorimétrie } \\
\left(\mu \mathrm{A} / \mathrm{cm}^{2}\right)\end{array}$ \\
\hline 13 & 45.08 & \multirow{5}{*}{0} & 0.1355 & 72 & 136 & 134 & 40.1 & 39.5 \\
\hline 28 & 45.08 & & 0.0881 & 53 & 102 & 97 & 40.8 & 38.8 \\
\hline 1 & 54.07 & & 0.0435 & 27 & 52 & 49 & 40.8 & 38.5 \\
\hline 31 & 45.08 & & 0.0655 & 42 & 78 & 77 & 39.4 & 38.9 \\
\hline 18 & 45.08 & & 0.0290 & 12 & 23 & 22 & 40.6 & 38.9 \\
\hline 2 & 54.07 & \multirow{5}{*}{1.70} & 0.1165 & 72 & 127 & 128.8 & 37.6 & 38.8 \\
\hline 22 & 45.08 & & 0.0620 & 53 & 96 & 89.8 & 38.6 & 36 \\
\hline 12 & 45.08 & & 0.0340 & 27 & 47 & 47.15 & 37 & 37.08 \\
\hline 25 & 45.08 & & 0.0545 & 42 & 75 & 71.02 & 38 & 35.9 \\
\hline 27 & 45.08 & & 0.0208 & 12 & 20.4 & 21.60 & 36.25 & 38.22 \\
\hline
\end{tabular}




\begin{tabular}{|c|c|c|c|c|c|c|c|c|}
\hline $\begin{array}{c}\mathbf{N}^{\circ} \\
\text { Eprou- } \\
\text { vette }\end{array}$ & $\begin{array}{l}\text { Surface } \\
\left(\mathrm{cm}^{2}\right)\end{array}$ & $\begin{array}{l}\text { Epais- } \\
\text { seur } \\
(\mu \mathrm{m})\end{array}$ & $\begin{array}{c}\text { Variation } \\
\text { de } \\
\text { masse } \\
\text { (g) }\end{array}$ & $\begin{array}{c}\text { Durée } \\
\text { d'attaque } \\
\text { t (heure) }\end{array}$ & $\begin{array}{l}\text { Dosage } \\
\text { Par A.A } \\
\text { (mg/L) }\end{array}$ & $\begin{array}{c}\text { Dosage } \\
\text { Par } \\
\text { Colorimé- } \\
\text { trie } \\
\text { (mg/L) }\end{array}$ & $\begin{array}{c}\mathbf{i}_{\text {cor }} \\
\text { Par } \\
\text { Absorption } \\
\text { atomique } \\
\left(\mu \mathrm{A} / \mathrm{cm}^{2}\right)\end{array}$ & $\begin{array}{c}\mathbf{i}_{\text {cor }} \\
\text { Par } \\
\text { Colorimétrie } \\
\left(\mu \mathrm{A} / \mathrm{cm}^{2}\right)\end{array}$ \\
\hline 15 & 45.08 & \multirow{5}{*}{2.25} & 0.558 & 53 & 72.8 & 67.3 & 29.2 & 26.96 \\
\hline 0 & 45.08 & & 0.0406 & 42 & 64.09 & 67.26 & 32.4 & 34 \\
\hline 19 & 45.08 & & 0.0265 & 27 & 35.7 & 35.60 & 28.10 & 28 \\
\hline 6 & 45.08 & & 0.988 & 72 & 104 & 91 & 30.88 & 27 \\
\hline 7 & 54.07 & & 0.0150 & 12 & 16 & 18.64 & 28.62 & 32.99 \\
\hline 10 & 45.08 & \multirow{5}{*}{2.59} & 0.0438 & 53 & 62 & 64.2 & 24.8 & 25.7 \\
\hline 5 & 45.08 & & 0.0316 & 42 & 46 & 44 & 23.25 & 22.24 \\
\hline 30 & 45.08 & & 0.0185 & 27 & 32 & 33 & 25.16 & 25.94 \\
\hline 21 & 45.08 & & 0.0096 & 12 & 13 & 14.6 & 22.9 & 25.8 \\
\hline 23 & 45.08 & & 0.0785 & 72 & 81 & 83.6 & 23.88 & 24.65 \\
\hline 32 & 45.08 & \multirow{5}{*}{3.08} & 0.0428 & 72 & 29 & 29.6 & 8.5 & 8.7 \\
\hline 20 & 45.08 & & 0.0263 & 53 & 21 & 20.4 & 8.4 & 8.1 \\
\hline 9 & 45.08 & & 0.0106 & 27 & 13 & 12.8 & 10.2 & 10.06 \\
\hline 8 & 45.08 & & 0.0182 & 42 & 20 & 19.6 & 10.1 & 9.9 \\
\hline 26 & 45.8 & & 0.0041 & 12 & 5 & 5.8 & 10.2 & 10.26 \\
\hline
\end{tabular}

\section{3-2. Etude électrochimique}

Le potentiel à l'abandon de l'électrode non phosphatée diminue au cours du temps et se stabilise après 50 minutes (Figure 3 ). Ce temps de maintien avant toute polarisation à tout essai est donc indispensable.

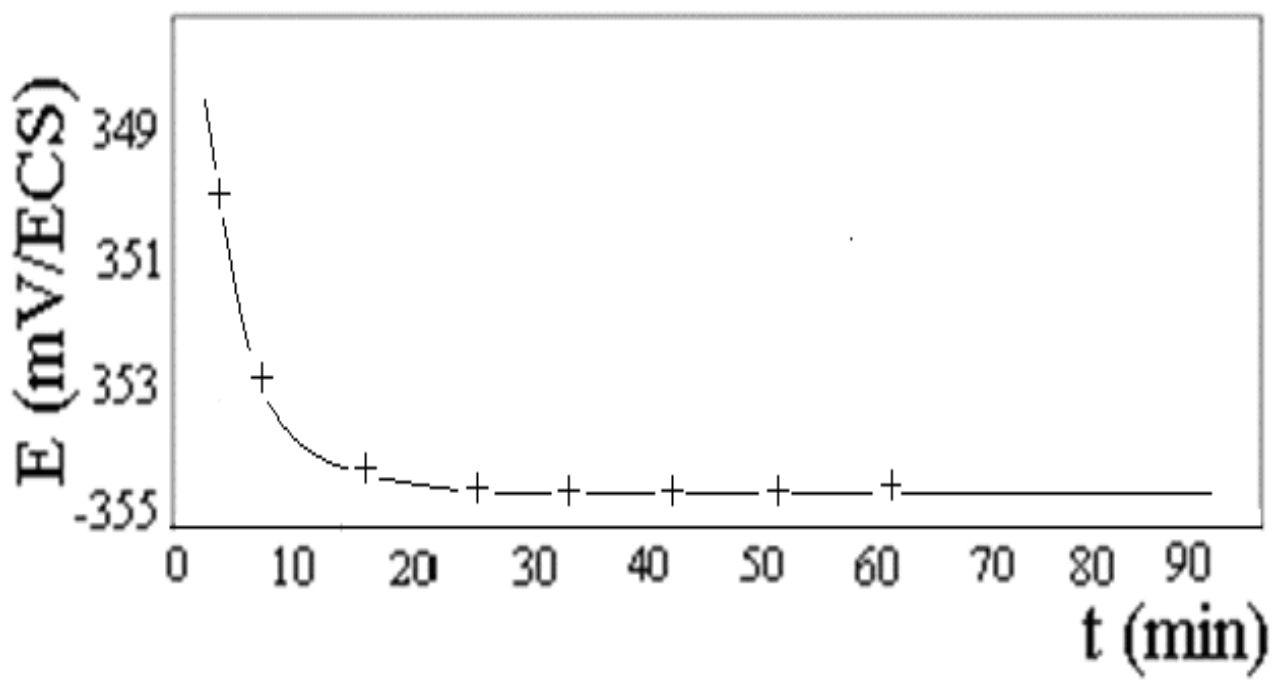

Figure 3 : Variation en fonction du temps pour $\Omega=1000 \mathrm{tr} / \mathrm{min}$ du potentiel de corrosion de l'électrode non phosphatée dans $\mathrm{NaCl} 3 \%$ 
La Figure 4 représente les courbes cathodiques potentiostatiques de l'électrode non phosphatée dans $\mathrm{NaCl} 3 \%$ à diverses vitesses de rotation. Elle montre que chaque courbe peut être scindée en deux parties:

- un premier domaine relatif à un palier de diffusion correspond à la densité de courant limite de diffusion provenant de la réduction de l'oxygène selon le bilan réactionnel :

$$
2 \mathrm{H}_{2} \mathrm{O}+\mathrm{O}_{2}+4 \mathrm{e}-\longrightarrow 4 \mathrm{OH}
$$

- le second domaine est observable vers les potentiels plus négatifs où se manifeste une croissance nette du courant correspondant à la réaction de réduction de l'eau suivant la réaction :

$$
2 \mathrm{H}_{2} \mathrm{O}+2 \mathrm{e}-\longrightarrow \mathrm{H}_{2}+2 \mathrm{OH}
$$

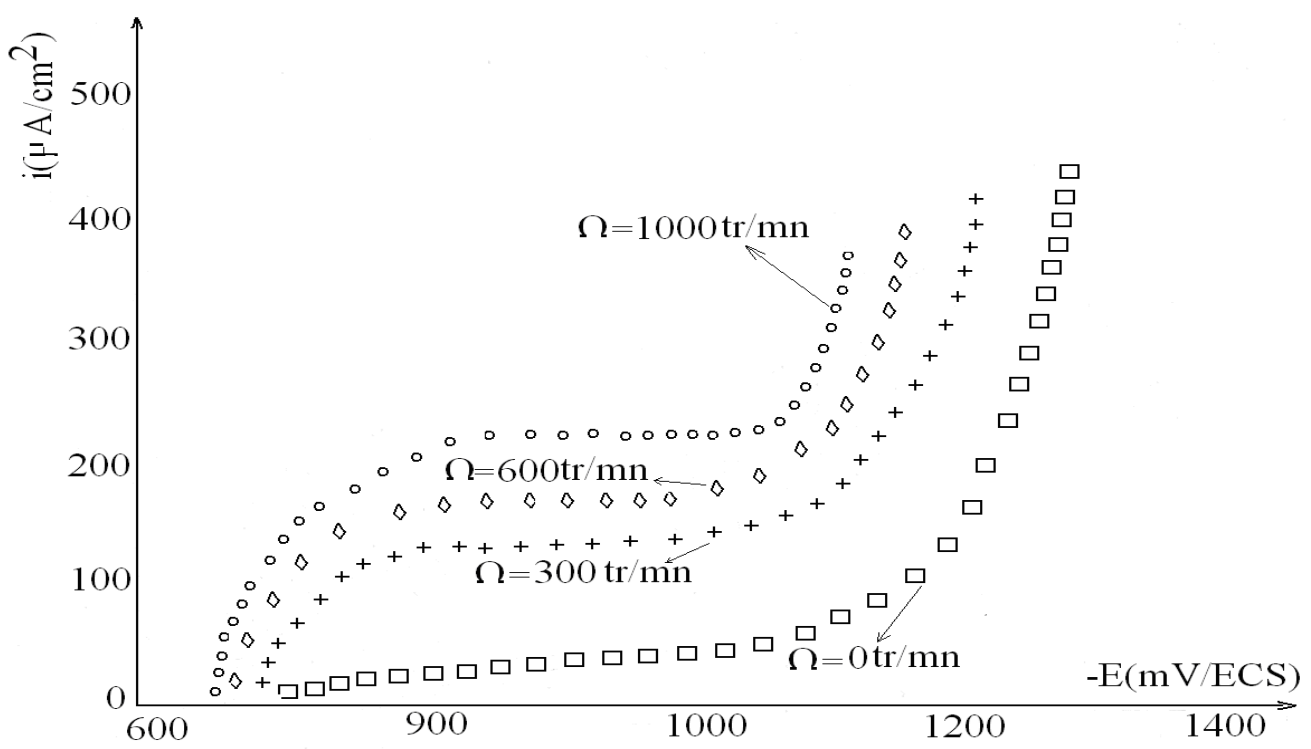

Figure 4 : Courbes potentiostatiques de polarisation cathodique de l'électrode non phosphatée dans $\mathrm{Na} C \mathrm{CL} 3 \%$ à diverses vitesses de rotation

Le changement des conditions hydrodynamiques se traduit par une augmentation de la hauteur du palier de diffusion avec l'accroissement de la vitesse de rotation.

Les différentes valeurs $i_{c o r}$ représentées en fonction de la racine carrée de la vitesse de rotation de l'électrode, se placent sur une droite qui ne passe pas par l'origine (Figure 5). Le décalage à l'origine a été expliqué par une diffusion à travers une couche poreuse de produits de corrosion [21,22]. 
La variation en fonction du temps du potentiel de corrosion libre, illustrée par la Figure 6, montre que ce dernier se stabilise au bout de quatre minutes, l'état quasistationnaire est rapidement atteint.

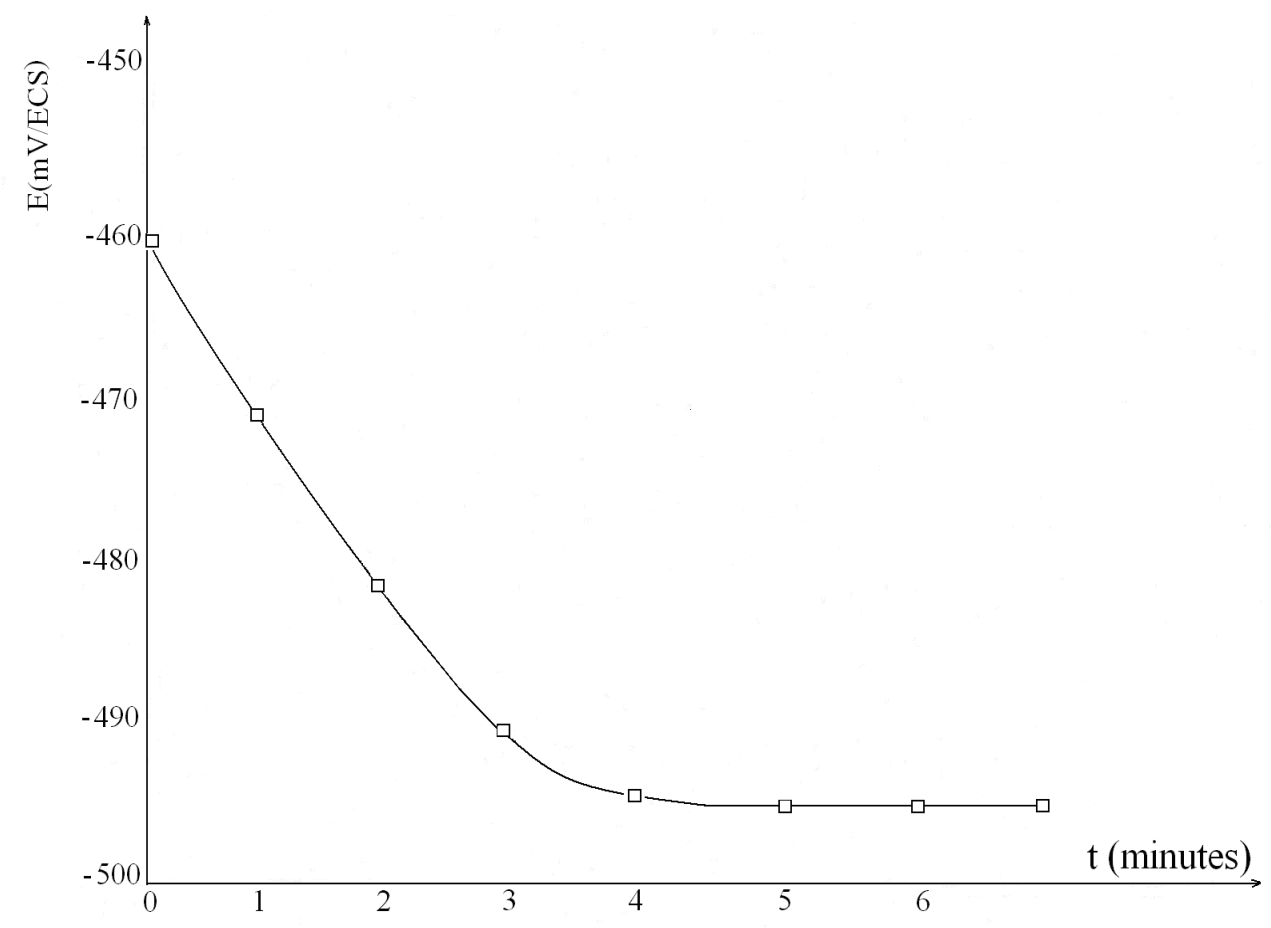

Figure 6 : Variation en fonction du temps pour $\Omega=1000 \mathrm{tr} / \mathrm{min}$ du potentiel de corrosion de l'électrode phosphatée dans $\mathrm{NaCl} 3 \%$

Les Figures $\mathbf{7}$ et $\boldsymbol{8}$ représentent les courbes de polarisation de l'acier phosphaté ; respectivement pour une vitesse de rotation de l'électrode de 0 et 1000 tours/minutes. On constate que l'allure de la courbe reste inchangée, un palier de diffusion suivi du domaine de réduction de l'eau.

Néanmoins, la hauteur du palier de diffusion diminue pour une épaisseur de la couche phosphatée supérieure à $2 \mu \mathrm{m}$ par rapport à l'électrode non phosphatée.

En effet, il prend les valeurs $38,30,25,10 \mu \mathrm{A} / \mathrm{cm}^{2}$ respectivement pour une épaisseur de la couche de $1.7-2.25-2.59$ et $3.08 \mu \mathrm{m}$.

D'autre part, l'augmentation de la vitesse de rotation de l'électrode conduit à l'augmentation de la hauteur du palier de diffusion. Ceci plaide en faveur de l'intervention d'un processus diffusionnel pur [23]. 


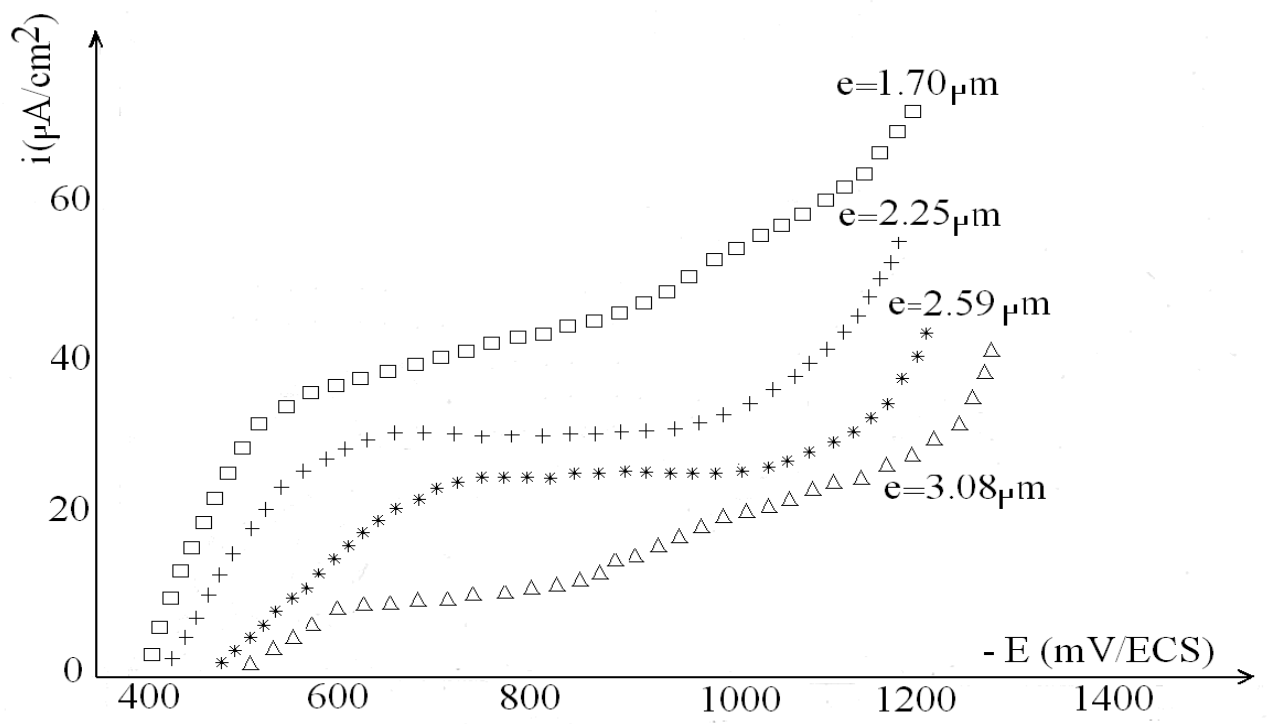

Figure 7 : Courbes potentiostatiques de polarisation cathodique de l'électrode phosphatée dans $\mathrm{NaCl} 3 \%$ pour diverses couches de phosphatation à $\Omega=0 \mathrm{tr} / \mathrm{min}$

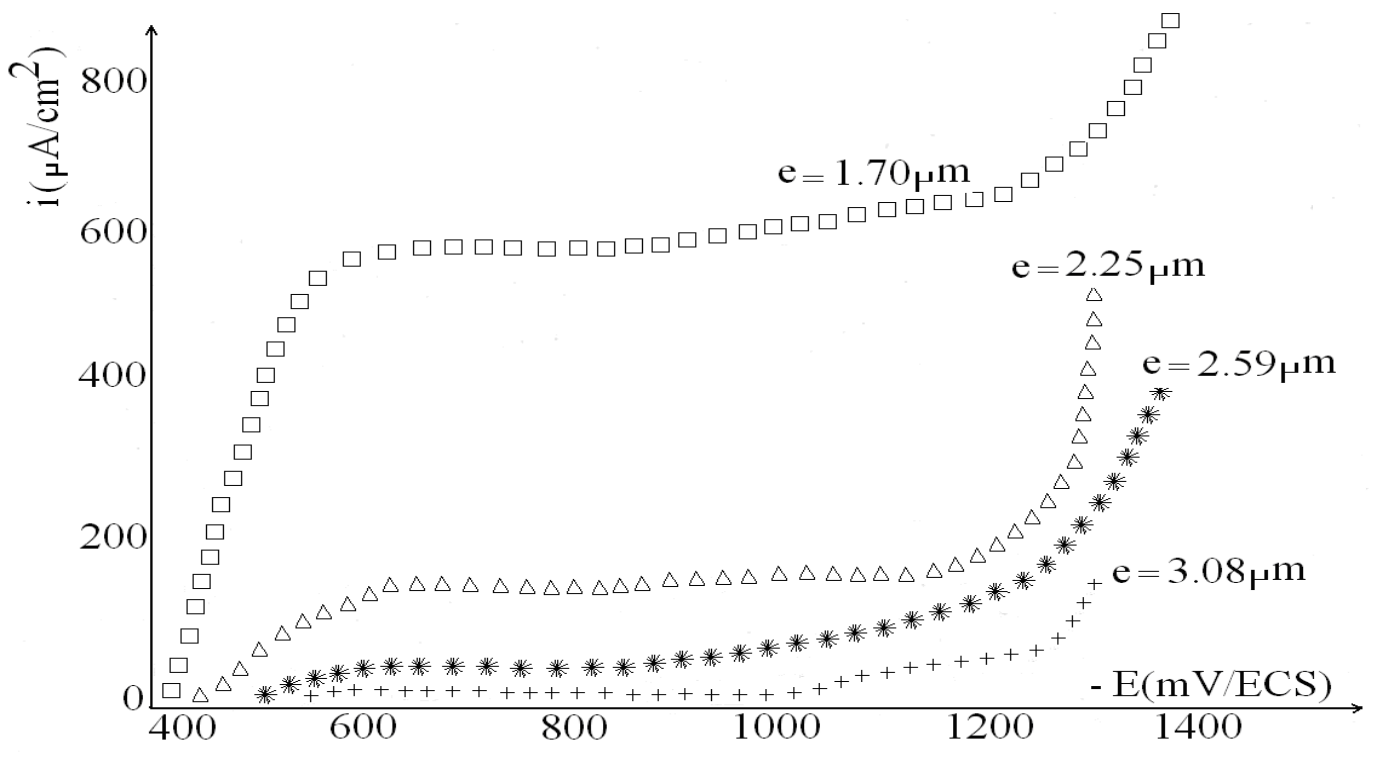

Figure 8 : Courbes potentiostatiques de polarisation cathodique de l'électrode phosphatée dans $\mathrm{NaCl} 3 \%$ pour diverses couches de phosphatation à $\Omega=1000 \mathrm{tr} / \mathrm{min}$ 
Le comportement anodique de l'acier revêtu dans le milieu d'attaque est résumé par les courbes de la Figure 9. On constate l'acier au carbone nu se caractérise par un comportement actif. La présence d'une couche de phosphate tend à transformer ce comportement passif, effet obtenu pour une épaisseur à $2 \mu \mathrm{m}$. Néanmoins, le potentiel de rupture de cette couche de phosphate reste encore très faible $(-230 \mathrm{mV} / \mathrm{ECS}$ pour $3.08 \mu \mathrm{m})$.

L'obtention de la couche de passivité augmente lorsque la concentration des ions chlorures croît comme en témoigne la Figure 10. De plus, la densité de courant de corrosion croît également avec la teneur en chlorures.

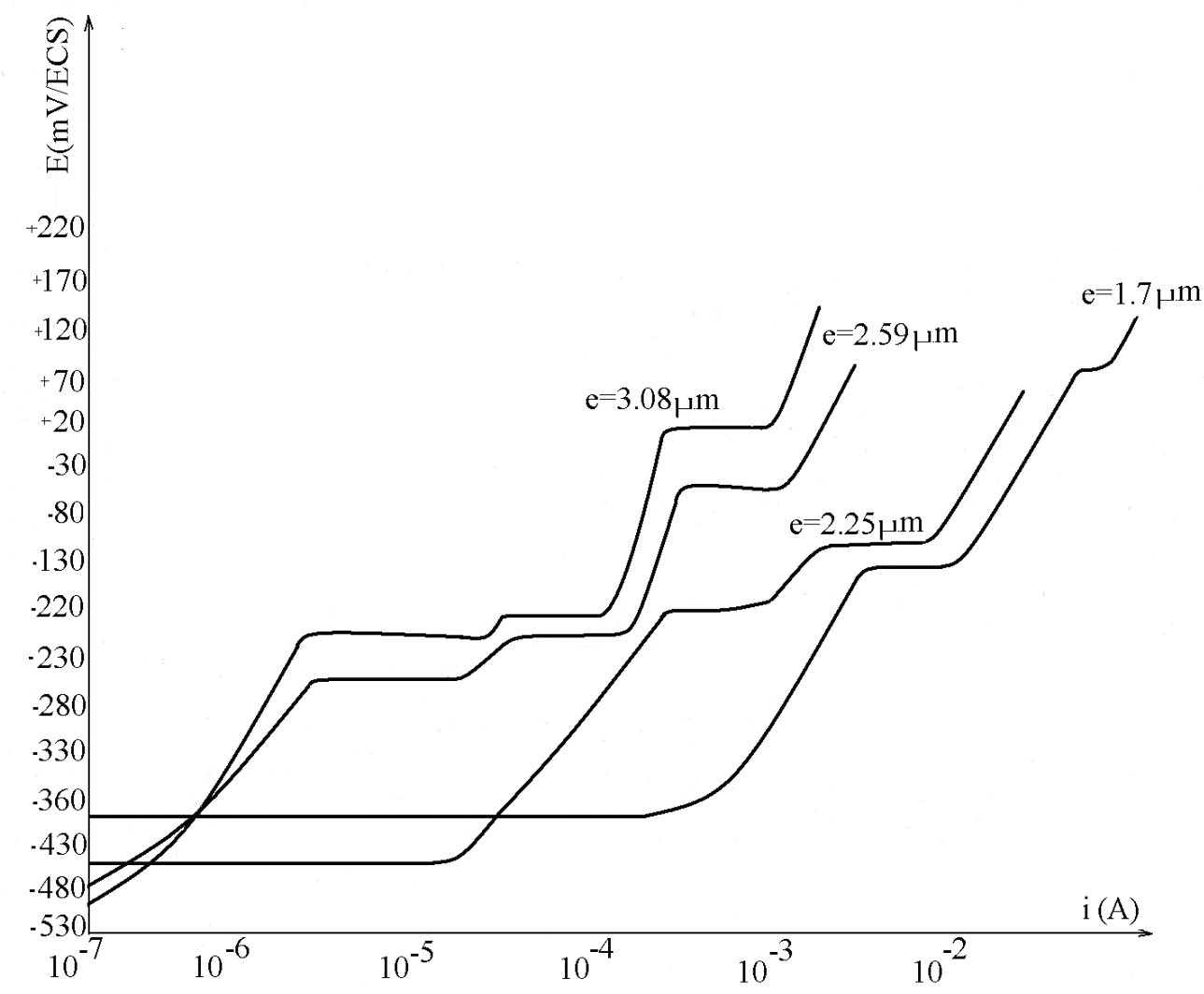

Figure 9 : Courbes potentiocinétiques de polarisation anodique à $\Omega=1000 \mathrm{tr} / \mathrm{min}$ de l'électrode phosphatée dans $\mathrm{NaCl} 3 \%$ à diverses épaisseurs de la couche de phosphatation 


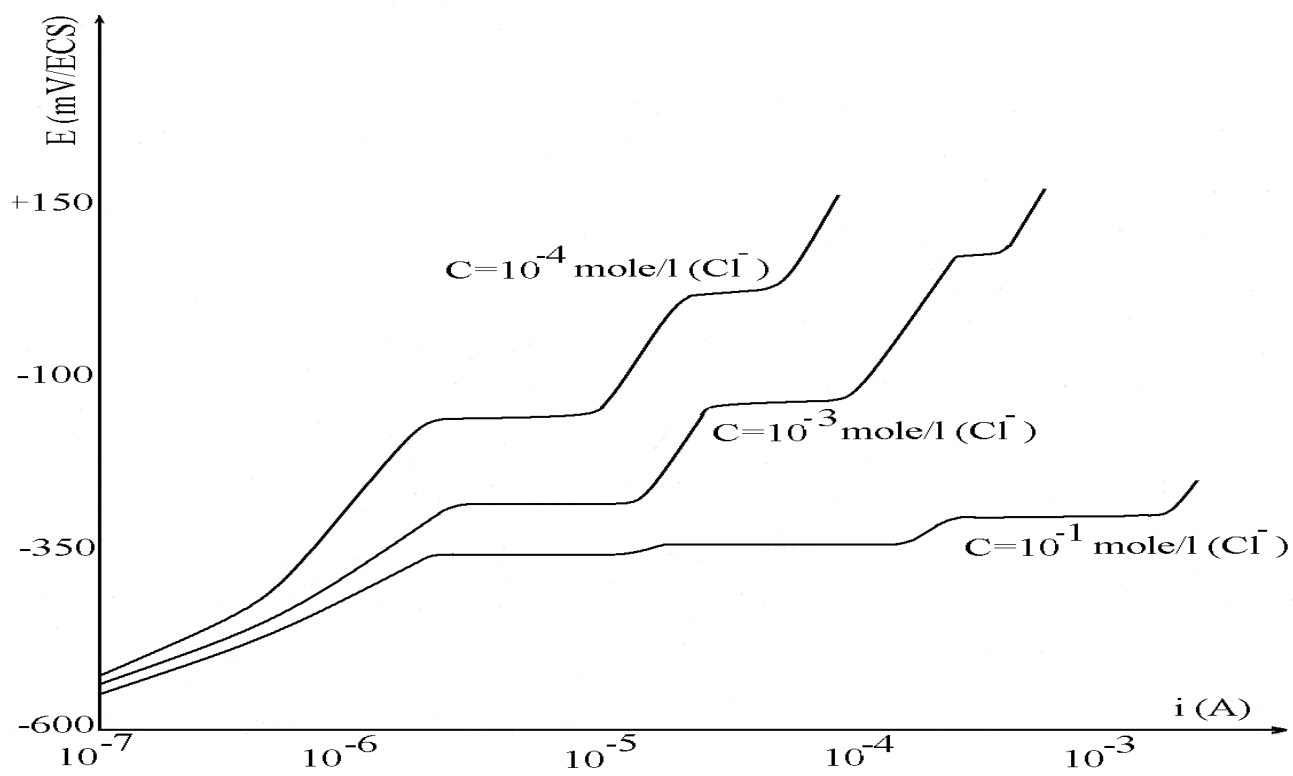

Figure 10 : Courbes potentiocinétiques de polarisation anodique de l'électrode phosphatée dans $\mathrm{NaCl} 3 \%$ à diverses concentrations en Cl à $\Omega=1000 \mathrm{tr} / \mathrm{min}$

\section{Conclusion}

L'allure des courbes cathodiques de l'acier au carbone dans $\mathrm{NaCl} 3 \%$ plaide en faveur d'un processus cinétique essentiellement contrôlé par une diffusion pure. Un palier de diffusion dont la hauteur est tributaire des conditions hydrodynamiques.

La phosphatation de l'électrode réduit le courant de corrosion par rapport à l'électrode non phosphatée dont la surface présente une hétérogénéité (rugosité, différence de structure cristalline,...), conduisant à de nombreux micro-éléments de piles. Cette hétérogénéité diminue par conversion chimique de surface, ce qui explique l'abaissement de la vitesse de corrosion au fur et à mesure que l'épaisseur de la couche de phosphatation croît. Ainsi, tend on vers un comportement passif pour une épaisseur de couche supérieure à $2.25 \mu \mathrm{m}$. Malgré les porosités de la couche de phosphatation, il semble que pour une épaisseur équivalente à $3 \mu \mathrm{m}$, l'acier revêtu présente une grande protection contre la corrosion dans $\mathrm{NaCl} 3 \%$.

Par ailleurs, les éprouvettes phosphatées $(\mathrm{e}=3 \mu \mathrm{m})$ et peintes, testées au brouillard salin pendant 500 heures, présentent une grande résistance à la corrosion confirmant la qualité protectrice pour l'épaisseur de $3 \mu \mathrm{m}$. Toutefois, il reste à caractériser la morphologie de la couche de phosphatation à cette épaisseur. D'autre part, l'augmentation du courant de corrosion avec la concentration en ions chlorures qui 
atteignent la surface métallique à travers les micropores de la couche de phosphate peut être expliquée en faisant appel aux mécanismes de la corrosion par piqûres.

Ce mécanisme se divise en deux parties, l'étape d'initiation selon laquelle, il y aura rupture du film de la couche protectrice, il s'ensuit un couplage galvanique entre la zone anodique restreinte et la zone cathodique favorisant l'étape de croissance des piqûres selon le bilan réactionnel :

- zone cathodique : $\mathrm{O}_{2}+2 \mathrm{H}_{2} \mathrm{O}+4 \mathrm{e}-\longrightarrow 4 \mathrm{OH}$

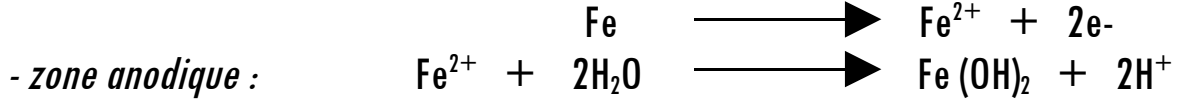

La formation locale d'un milieu acide favorise la propagation durant laquelle la dissolution du fer à l'état actif et l'acidification du milieu s'entretiennent mutuellement (mécanisme auto catalysé). Par la suite, la vitesse de corrosion croît au fur et à mesure que la concentration des ions chlorures augmente.

L'ensemble des résultats obtenus dans ce travail a permis de mieux cerner les phénomènes de corrosion d'un acier au carbone phosphaté dans une solution de chlorure de sodium $3 \%$. Nous avons dans un premier temps, montré pour un acier non phosphaté à l'aide du couplage des méthodes électrochimiques et des méthodes de la détermination directe de la quantité du fer passé en solution, que la cinétique de la réduction de l'oxygène dissous est du type diffusionnel. Dans un deuxième temps, nous nous sommes intéressés à l'étude du comportement électrochimique du même acier, mais phosphaté dans $\mathrm{NaCl} 3 \%$.

Les essais gravimétriques ainsi effectués ont montré que l'effet de l'épaisseur de la couche de phosphatation a une influence importante sur la résistance à la corrosion de l'acier utilisé. Le test au brouillard salin, montre une différence d'attaque appréciable sur les différentes éprouvettes à diverses épaisseurs de la couche de phosphatation, ce qui est en accord avec le résultat précédent.

D'autre part, les essais électrochimiques ont également confirmé cet état de fait par une diminution de courant de corrosion quand l'épaisseur de la couche croît. L'éprouvette phosphatée $(3 \mu \mathrm{m})$ et peinte, présente une grande résistance à la corrosion.

\section{Références}

[1] - Fetzer, Karl; Pfiz, Roland; Strube, Gernot. "Method for coating components consisting of hardened steel or cast iron with a zinc-nickel', PCT Ind. Appl (2002), $19 \mathrm{pp}$.

[2] - L. Bazzi, R. Salghi, Zine, S. El Issami, S. Kertit, Hammouti "Corrosion inhibition of 6063 aluminium alloy by means of the inorganic composition in a solution of 3\% solution chloride", Canadian Journal of Chemistry 80 (1) (2002) 106-112. 
[3] - Hadji Ali, Lemmoui Abdenacer, M. Romanov Lev rt Y. Kozlov Leonid. "Behaviour of alloyed white cast-iron in a corrosive environment". Annals de chimie (Paris) 25(4) (2000) 307-316.

[4] - "Chemical treatment of surfaces" - Technical guide for the process industries November (2002)

[5] - THEOPHILE GHEGUEN, Techniques de l'Ingénieur - Journées d'étude sur les peintures en poudres - pp12-23 (1989)

[6] - M. DUPRAT, Thèse de Doctorat d'Etat à INP Toulouse France -(1981) pp 35-78

[7] - PATRICK GOUGELIN, Thèse de $3^{\text {ime }}$ Cycle Paris 6 - (1985) pp 15-28

[8] - O.D ; RENEDO, J.A. MARTENEZ - Journées d'Electrochimie, Toulouse, (1999)

[9] - G.D. CHEEVER, Journal of Paint Technology, 39 (1967) I

[10] - N. AUGER, F. CARROIS, "Caractérisation des plaquettes artificielles de simulation représentatives de l'altération des ferreux archéologiques", $3^{\text {ème }}$ année ISITEM Nantes, Janvier (2000).

[11] - W. MACHU, Mettalwirtschaft, 22 (1943) 481

[12] - W.I. WULFSON and RABINOVICH, Korrosiya I. Borbasnei, 3(1937) 363)

[13] - V. CUPR et J.B. PELIKAN, mélloberfläche, Juin-Juillet(1965)

[14] - Fetzer, Karl; Pfiz, Roland; Strube, Germot - Method for coating components consisting of Hardened steel or cast iron with a zinc-nickel alloy", PCT Int. Appl. Germany (2002)

[15] - W. MACHU, Die Phophatierung, Verlag Chemie, Berlin (1950)

[16] - R. Norling, T. Olefjord - "Erosion-corrosion of Fe-and Ni-based alloys at $550^{\circ} \mathrm{C}$ "- Wear 254 (2003) $173-184$

[17] - M. GEBHARDT, Galvanotechnik, Oberflächenschutz, Zirich, (1966)

[18] - Hans Berns - Comarison of wear resistant MMC and white cast iron-Wear 254 (2003) 47-54

[19] - Hans Berns, Comparison of wear resistant MMC and white cast iron, Wear 254 (2003) 47-54

[20] - T.S.N.S. NARAYANNAN European Coatings Journal, $N^{\circ} .4$ (1999) 138-141

[21] - Abdelouahab. ETTAQI, Abdellatif. IRHZO et Mustapha. ZERTOUBI, " Etude de l'influence de la teneur en chrome sur la résistance à la corrosion des fontes (pompes d'extraction) dans des solutions de sulfate de cuivre et de sulfate de fer acidifiées [extraction de cuivre - mine de cuivre] II, BULLETIN OF ELECTROCHEMISTRY (BOE), Vol.21, $\mathrm{N}^{\circ} 3$, Mars (2005)

[22] - Abdelouahab ETTAQI, Abdellatif IRHZO et Mustapha ZERTOUBI, "Influence d'addition des substances inhibitrices à base de soufre et de phosphore sur le comportement électrochimique d'une fonte fortement alliée au chrome dans un milieu sulfurique composé de $\mathrm{FeSO}_{4}$ et CUSO 4 II, BULLETIN OF ELECTROCHEMISTRY, (BOE), Vol.21, Nº, Avril (2005)

[23] - A. ETTAQI, A. IRHZO, R. DRISSI DAOUDI et M. ZERTOUBI, II influence de l'addition d'inhibiteurs azotés sur le comportement électrochimique et métallurgique de fontes au chrome - nickel (pompes d'extraction) en milieu acide (mine de cuivre) II, Afrique SCIENCE (ISSN 1813-548X), Vol.01, N 1, Janvier (2005) 31-53 